\title{
Transition between different UK mental health services - young people's experiences on what makes a difference.
}

\author{
Cathy Street*, Leanne Walker, Amanda Tuffrey, Anna Wilson \\ Great Involvement Future Thinking (GIFT) Partnership, UK
}

\begin{abstract}
There is longstanding and widespread concern about the poor care offered to young people at the point of making a transition from mental health services for children and young people to services for adults. Research has highlighted that many experience sub-optimal care including poor planning, little information, limited choices and a sense of the whole process being rushed. A number of national UK government reviews have been convened over the last fifteen plus years that have raised poor mental health transitions from children and young people's mental health services (CYPMHS) as an area requiring urgent attention; this includes Future in Mind. Promoting, protecting and improving our children and young people's mental health (Department of Health and NHS England) which sets out the national vision for children and young people's mental health in the UK. Young people report feeling excluded from important decisions about their care, that transition from CYPMHS is often rushed, poorly planned and that their wishes are disregarded. This article describes some of the policy background to this issue and the service improvement initiatives that have emerged in the $U K$ in response including a new contractual indicator to try and incentivize service change. At the centre of this, the article presents the perspectives and transition experiences of a number of young people who are part of GIFT, Great Involvement Future Thinking, a partnership between a mental health consultants, researchers and trainers and young people who work as sessional workers and national advisors, drawn from across the UK, all with experience of using NHS mental health services. Three of these young people, Leanne, Amanda and Anna co-authored this article and provide vivid examples of the barriers many young people in transition can face, along with ideas as to what would improve mental health service transitions.
\end{abstract}

Keywords: Transition, Adulthood, Geographic area, Mental health.

Accepted on January 23, 2018

\section{Introduction}

Young people's views about health services are recognized as an important dimension in understanding whether services are working well or need adaptation. In the field of mental health, in particular, involving young people in the development, or review, of the services they use, has been a key part of government policy aimed at improving services. Service user involvement, of children, young people, parents and carers, was a central plank of the Children and Young People's Improving Access to Psychological Therapies (CYP IAPT) programme which aims to transform CYPMHS through the embedding of evidence-based practice across services (achieved through a large scale programme of workforce training) and supporting new ways of working, including partnerships between NHS mental health service providers and voluntary and community sector (VCS) partners.

GIFT - Great Involvement Future Thinking - is a partnership between a small group of mental health consultants, researchers and trainers working collaboratively with a group of young people drawn from across the UK, all with experience of using NHS mental health services. GIFT was formed in 2012 to support young people's participation in the CYP IAPT programme.
GIFT 'young sessional workers' were involved in a wide range of activities including speaking at conferences or running workshops aimed at improving clinical practice in mental health services, reviewing local mental health services, developing new web-based resources, supporting local participation groups and sitting on the various national advisory groups that steered the delivery of CYP IAPT.

Transition from mental health services for children and young people was identified by the GIFT young sessional workers as an area where they wished to develop new resources to improve practice and/or to raise awareness of the problems many young people were encountering. That they identified this issue is not surprising given the context of widespread concern about some groups of young people experiencing problems accessing mental health support or falling 'between the gaps' of different services due to different referral criteria, inconsistent age thresholds or a lack of provision for some groups identified as 'vulnerable' in Future in Mind $[1,2]$. A lack of appropriate services for young people in the 16-25 age range, that is the age range during which transition is most likely to take place, was also being highlighted $[3,4]$, with a variety of reports noting that this situation was worsening due to service cutbacks, in particular to VCS counselling and other support provision for young people $[5,6]$. 


\section{Materials and Methods}

Some of GIFT's young sessional workers, Leanne, Amanda and Anna have co-authored this article with one of GIFT's cofounders. The article draws on a brief review of key research and UK government policy concerning young people's mental health transitions, however, the main focus is young people's views of care and support during the transition phase. In the sections that follow, these three young people draw on their own experiences and those of their peers, of using mental health services, both those for children and young people and those for adults and including across the transition phase of care, to explore some of the difficulties young people can experience in making this important journey across provision. The article concludes with their suggestions and ideas of what would make a difference.

\section{The Challenge of Improving Care and Support at the Time of Transition}

Transition is the term used to describe the process of moving someone from one health service to another; it refers to the coordination, planning and preparation for supporting a person to leave one service and begin attendance at another. In the field of child and adolescent mental health, transition from services for children and young people to adult mental health services (AMHS) typically happens at the age of eighteen. Poor and disjointed care at this significant time point, crucially leaving young people with mental health difficulties unsupported, some falling through a gap of inconsistent service referral criteria or being left having to navigate their own way through complicated service arrangements, has been an area of significant concern in the UK over at least the last fifteen years. This has resulted in a variety of government policy initiatives and plans to transform Children and Young People's Mental Health Services (CYPMHS), previously known as Child and Adolescent Mental Health Services (CAMHS).

Why improving transition is recognized as important is clearly illustrated by the following statement. Taken from good practice guidance for the provision of psychiatric services to adolescents/young adults produced by the interfaculty working group of the Child and Adolescent Faculty and the General and Community Faculty of the Royal College of Psychiatrists in 2008, this notes: "The transition from adolescence to adulthood is a crucial stage of social, personal and emotional development. It coincides with the emergence of personality disorders and a steep rise in the rates of mental disorder..." [7]. A more recent Royal College of Psychiatrists faculty report Good mental health services for young people [2], makes similar points. This highlights that young people and families report that they find the change in service philosophy between mental health services for children and young people and those for adults confusing, and that there is a lack of services for a number of specific groups including young people with attention-deficit hyperactivity disorder (ADHD) and emerging personality disorders.

Examples of the concern surrounding poor transitional care include that it has been an issue raised in a number of House of Commons Select Committee on Health reports (for example, the
Fourth Report of the HC for Session 1999-2000 [8] Transitions between child/adolescent and adult services), was the subject of a report Bridging the Gaps: Health Care for Adolescents by the Royal College of Paediatrics and Child Health in 2003 [9] and was a central theme in Children and young people in mind: the final report of a National CAMHS Review [10]. This noted the following: "During our practice visits, it was the transition from CAMHS to adult mental health services that causes children, their families and service providers most concern. These problems are not new and they relate to the considerable cultural and service criteria differences between the two broad groups of services."

The ongoing and persisting concern about transition is evident in a number of more recent important documents, notably Future in Mind. Promoting, protecting and improving our children and young people's mental health and wellbeing [1]. This sets out the national vision for children and young people's mental health, documents the case for change and details how to make this happen, which includes a section focused on managing transition. Then in 2016, guidelines on young people's transition were published by NICE, the National Institute of Health and Care Excellence [16]; these emphasise the importance of planning, of involving the young person and taking full account of their views and needs and recommend that young people should have a 'named worker' to coordinate their transition care and support. Lastly, in 2017, NHS England introduced a new CQUIN - a Commissioning for Quality and Innovation contractual lever intended to drive transformational change and "incentivise improvements to the experience and outcomes of young people as they transition out of Children and Young People's Mental Health Services [17]."

In the health research field, the poor transition experiences of young people using mental health services, has been an area attracting attention, and indeed, the TRACK study [11] is cited in NHS England information about the rationale for introducing the transition CQUIN. TRACK identified four 'optimal' markers of transition - good information transfer; a period of parallel care (when both CYPMHS and adult services would work together); planning, including the holding of a meeting involving the young person and professionals for both services for children/young people and services for adults and continuity of care. However, findings from the study also indicated that, based on studying 154 young people over a 1 year period, less than $5 \%$ of these young people experienced all four of the markers of optimal transitional care.

\section{Results}

\section{Young people's perspectives and experiences}

Young people's experiences of transition provide an important dimension on understanding both what works, and more crucially, what often does not work at the point of leaving mental health services for children and young people. These data not only shed light on the many barriers they may encounter in trying to move to an adult service but also, highlight both some of the more subtle cultural differences between services that can impede transition - how there can often be a disconnect between 
policy recommendations and guidance and 'real life' practice. Young people's suggestions of what would make a difference also provide some practical solutions to the barriers identified.

The young people from GIFT who contributed to this article all had experience of using CYPMHS and some had experience of making a transition to adult mental health services or experienced the barriers just described. A number were involved in the consultation for the afore-mentioned 2016 NICE guidelines on transition and were members of the Children and Young People's Mental Health and Wellbeing Taskforce that produced Future in Mind [1].

In the main, their experiences of transition were poor, largely because the process was marked by a lack of planning, little preparation, inadequate information and then finally, a move that seemed abrupt. Furthermore, these young people talked of feeling largely left out of any discussions about the proposed transition with one young person describing being involved in a transition meeting but at the point of decisions being made, being asked to leave the room. Amanda commented:

"I would have liked to have known the full meeting agenda, who the people were, what services they came from and what help they could offer me. Instead, I felt put on the spot to talk to a room full of strangers about deeply personal issues...this made me feel marginalised, hopeless and helpless..."

This particular example illustrates that even when some things are in place to support a transition, that is, there is evidence of services following good practice guidance, NICE guidelines and/or implementing the 'optimal markers' identified, the reality of how young people experienced this, can be far from helpful.

Both Leanne and Anna described a particular problem resulting from their transition coinciding with them leaving home to go away to university. This move to another geographic area, it seemed, made it impossible for services to join up and plan collaboratively. Furthermore, Leanne and Anna also experienced being told they were 'not ill enough' to require transition or to meet the referral criteria for adult mental health services, a situation that has been reported on in a number of reports raising concerns about increasingly high mental health service thresholds $[12,13]$.

This issue of the problem of service co-ordination across different areas at the point of transition is well illustrated through Leanne's reflections on her move to university:

"I still needed some support and wasn't sure where that was going to come from after being told I wouldn't meet the criteria for adults. I wasn't moving far but it was out of the area I was currently living in and that presented further problems. In the end the (mild) problems I was experiencing worsened - then I was accepted into a service. You shouldn't have to 'get worse' in order to continue your progression. Moving to university is challenging enough, without losing the support you already have."

Anna expands on this, noting:

"My experience was very similar to this, being told that I was not "ill enough to meet criteria for adult services". Telling a young person that adult services won't take them has more wide spreading consequences than a clinician might think. It fed into the hopelessness I felt with the depression and anxiety I experienced when I moved to university, initially stopping me from seeking help as I believed no services would see me as ill enough. It also reinforced the common irrational beliefs I had - that I wasn't that ill and just lazy, and that I should be able to cope fine on my own."

The confidence of staff to support young people through a transition to adult mental health services was another key issue raised. For example, these and other GIFT young sessional workers described experiencing what they termed 'crossed wires' between different professionals who held different views about the same situation and also a general failure for anyone to take charge of the transition planning, this often resulting in a failure to follow through on anything that what agreed. Another problem was encountering staff in adult services who seemed to know nothing about the young person's situation or needs, of having to constantly re-tell their story, or of having to prove their need for mental health care; Leanne noted:

"When I finally got to adult services, the service was poor, they clearly hadn't read my history and I had to tell my whole life story over again. That's hard".

Crucially, how these experiences left this group of young people feeling was: guilty - that they were putting pressure on their families; scared - of the unknown and of being left without any support; angry - that their views and wishes were not being heard or were disregarded, and powerless - that they appeared to have no say in any of the decisions. Hardly surprising therefore, that a sizeable number of young people disengage at services at the transition point, or report finding themselves in services that do not appear to fit with their needs [14].

\section{What they would have liked?}

A plan that was built to match their individual needs, being involved in the process and to have been offered some choices, were identified by GIFT young sessional worker as the three priorities in improving young people's transitions. Accompany this were the following: that young people need information about adult services and what to expect (acknowledging that these services are likely to be different); that information about alternatives to NHS mental health services should also be provided - for example, about counselling and other support provision that may be available in colleges and universities as well as those offered by the charity sector. A very useful insight into how other services (other than NHS adult services) are presented to young people also emerges from the following reflections from Anna:

"From my experience clinicians rarely speak about non-NHS services or express negative views of them. It would be great if CAMHS acknowledged and discussed with YP alternative services. For example crisis helplines can be invaluable out of hours and some charities can offer different types and lengths of counselling with less restrictive criteria than the NHS which can be perfect for people like me that "fell through the gap". 
Furthermore, Amanda described this creating unnecessary anxiety and uncertainty, noting:

"Scaremongering from children's services about different thresholds and criteria created a self-fulfilling prophecy resulting in my illness worsening and becoming more entrenched..."

It was emphasised that young people need to be given time to prepare for a move and that decisions should not be left until the last minute. It should also not be left up to young people to be the ones sharing information about their needs or to find services themselves. Anna described how:

"Small things like the your clinician taking time to find the services available if your moving location can really show they care and want the best for you."

Leanne suggested that services should make better use of the NHS England Children and Young Person's Mental Health Service Information Passport [15], which aims to help service users to "own and communicate their story when moving between different services" [15]. The value of using this tool is illustrated by the following comment from Leanne:

"When I was given the opportunity to create a mental health passport, a concise picture of my circumstances and information in my own words, I was relieved I had given the voice back to the service user..."

Crucially, it was also pointed out that mental health professionals should "not make assumptions about what a young person needs or that the move between different mental health services is the only transition facing a young person... you need to ask."

\section{Discussion}

The perspectives and firsthand experiences of the young people presented in this paper provide clear evidence that poor transitional care can have both immediate and long-term adverse consequences, with young people feeling disempowered, excluded from decision-making processes - and that crucially, the barriers they face can actually exacerbate some of the difficulties they experience as a result of their mental health difficulties. In addition to their own direct use of services, including at the point of transition, Amanda, Anna and Leanne, through their extensive work for GIFT, alongside other service development activities for NHS England, Health Education England, NICE, as well as a variety of local NHS CYPMHS, provide an important national insight into these issues.

Their suggestions for what would make a difference clearly resonate with much of the good practice guidance for health services, notably that there should be good information transfer, co-ordination of different services, planning that involves the user of the services in question. Some mention is also made of tools that have been created to support these processes but which it appears, are sadly not in use in many NHS services.

The high level of policy interest and activity on improving young people's mental health transitions is welcome; however, it is too early to tell if the introduction by NHS England of
Transitions out of CYPMH CQUIN will bring improvements in the support, planning and co-ordination of care and treatment at the juncture.

Amanda, Anna and Leanne who co-authored this article described three underpinning principles. Firstly, a 'good' transition empowers young people by putting them at the centre of the process and takes account of what is important to them. Secondly, it informs in that the process is based on sharing information including across different agencies and considers options with the young person right from the start. Thirdly, it supports young people to have the confidence to engage with a new service and to move on with their lives. Anna highlighted:

"These principles highlight the importance of seeing every YP as an individual, with people have differing wants and well as needs; through this time of national interest it is important to remember that with transition a one size fits all approach is not appropriate"

Following these principles and embedding them in the way mental health practitioners work with young people, they concluded, would make a significant difference to improving the experience of transition for all young people who need to make a service move.

\section{Conclusion}

This article has provided an overview of the concerns that surround children and young people's transition from mental health services, including some of the key policy drivers to improving this situation. The various policy and research reports cited provide clear evidence that poor co-ordination, planning and continuity of care at this critical point in a young person's use of services, is a longstanding worry. Furthermore, despite good practice guidance and the development of tools to aid information sharing, it appears from the experiences described by three young co-authors, that these difficulties persist. As noted, there are now NICE guidelines in place [16] and a CQUIN on young people's transitions out of CYPMHS has been introduced [17]. It is to be hoped that these may provide important levers in improving practice in the years ahead.

\section{References}

1. Department of Health and NHS, England. Future in mind - Promoting, protecting and improving our children and young people's mental health and well-being. 2015.

2. Royal College of Psychiatrists. Good mental health services for young people FR/CAP/GAP/01. 2017.

3. Pona I, Royston S, Bracey C, et al. Seriously awkward. How vulnerable 16 and 17 year olds are falling through the cracks. The children's society. 2015.

4. Paul Hamlyn Foundation and Mental Health Foundation. Literature review summary version: Young people aged 16 to 25: The promotion of mental health and well-being and the early intervention in mental health problems. 2008.

5. Youth Access. Picking up the pieces: Results of a survey on the state of young people's advice, counselling and support 
services, London. The Young People's Health Partnership. 2013.

6. Youth Access. Making integration a reality. Part 2: Developing effective holistic services for young people in transition. London. The Young People's Health Partnership. 2014.

7. Lamb C, Hall D, Kelvin R, et al. Working at the CAMHS/ adult interface: Good practice guidance for the provision of psychiatric services to adolescents/young adults. Royal College of Psychiatrists. 2008.

8. http://www.parliament.uk

9. Royal College of Paediatrics and Child Health. Bridging the gaps: Healthcare for adolescents RCPCH. 2003.

10. Department of Health and Department for Children Schools and Families Children and Young. People in mind: The final report of the National CAMHS Review. 2008.

11. Singh S, Paul M, Ford T, et al. Transitions of care from child and adolescent mental health services to adult mental health services (TRACK study). A study of protocols in Greater London. BMC Health Serv Res. 2008;8:135.

12. Frith E. Access and waiting times in children and young people's mental health services. Education Policy Institute. 2017.

13. Social Care Institute for Excellence (SCIE). Improving mental health support for our children and young people. Expert Working Group Final Report. 2017.

14. http://www.rcpsych.ac.uk

15. NHS England. Children and young person's mental health service information passport. 2015.

16. NICE. Transition from children's to adults' services for young people using health or social care services. NICE Guideline. 2016.

17. NHS England. Transitions out of children and young people's mental health services (CYPMHS) CQUIN. 2017.

\section{*Correspondence to:}

Cathy Street

Great Involvement Future Thinking (GIFT)

Partnership

UK

Tel: $+0044-7775892280$

E-mail: cathy.street1@btinternet.com 